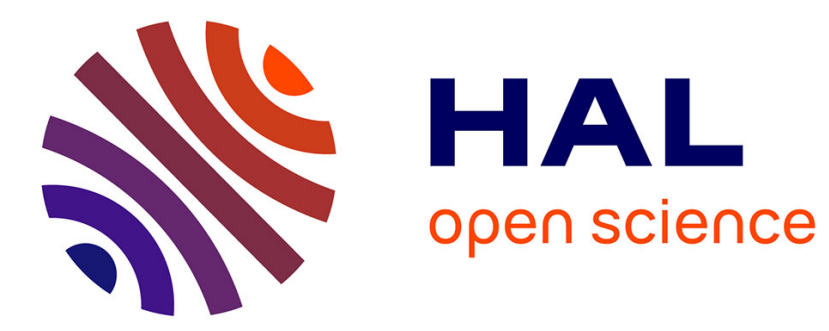

\title{
An Energy Based Approach for Passive Dual-user Haptic Training Systems
}

Fei Liu, Arnaud Lelevé, Damien Eberard, Tanneguy Redarce

\section{To cite this version:}

Fei Liu, Arnaud Lelevé, Damien Eberard, Tanneguy Redarce. An Energy Based Approach for Passive Dual-user Haptic Training Systems. 2016 IEEE IROS, Oct 2016, Daejeon, South Korea. 10.1109/IROS.2016.7759771 . hal-01340714

\section{HAL Id: hal-01340714 https://hal.science/hal-01340714}

Submitted on 7 Jan 2019

HAL is a multi-disciplinary open access archive for the deposit and dissemination of scientific research documents, whether they are published or not. The documents may come from teaching and research institutions in France or abroad, or from public or private research centers.
L'archive ouverte pluridisciplinaire HAL, est destinée au dépôt et à la diffusion de documents scientifiques de niveau recherche, publiés ou non, émanant des établissements d'enseignement et de recherche français ou étrangers, des laboratoires publics ou privés. 


\title{
An Energy Based Approach for Passive Dual-user Haptic Training Systems
}

\author{
Fei Liu, Arnaud Lelevé, Damien Eberard and Tanneguy Redarce
}

\begin{abstract}
This paper introduces a new controller for dualuser training systems, designed by way of an energy based approach. Dual-user training systems are useful for supervised hands-on training when a trainer shows the right gestures to a trainee and where the forces to apply on the tools are difficult to dose. An energy shared control (ESC) based architecture is proposed, based on an intrinsically passive authority sharing mechanism which is enhanced to provide a full force feedback to both users. As this enhancement may violate the natural passivity of the system, a passivity controller is introduced. A task based comparative study with two other dual-user schemes (Complementary Linear Combination (CLC) and Masters Correspondence with Environment Transfer (MECT) from [1] is conducted, which reveals analogous performances. Real-time experiments demonstrate good tracking performances.
\end{abstract}

\section{INTRODUCTION}

Medical staffs require continuing hands-on training on ever evolving medical methods. During their education, they usually train on black boxes, cadavers or animals, and more recently on passive and active simulators, before training on real patients. It has been proven that computer based haptic training simulators lead to an efficient training on advanced tasks (see [2]). In the context of this paper, their only drawback is that, in general, the trainee is alone in front of his simulator and cannot take benefit of a supervised training. Hence, in supervised hands-on training, the trainer takes the hands of the trainee in his own hands in order to guide him and to perform difficult gestures. While the hands' motion is driven by the trainer, they both share the haptic feedback derived from the manipulated tools. Therefore it is difficult for the trainer to dose his forces and for the trainee to feel the right level of forces to apply. Dual-user systems permit that each one directly manipulates a different master haptic interface acting as a common fake tool while the real tool is actuated by the slave part of the system. This slave part can also be a virtual tool in a virtual environment.

Introduced by Nudehi et al. in [3], some variations of dualuser system have been studied in [1], [4], [5]. These systems provide a haptic interface for both users (two masters) and a software architecture which connects these devices to perform a combined control over a slave system. The common concept is that the interfaces provide force feedback to both master users (trainer and trainee) according to a dominance factor $(\alpha \in[0,1])$. When $\alpha=1$ (resp. 0$)$, the

The authors work Univ. Lyon, INSA Lyon, Laboratoire Ampère (UMR 5005) F-69621, Lyon, France (Emails: ffei.liu, arnaud.leleve, damien.eberard, tanneguy.redarce\}@insa-lyon.fr).

The authors acknowledge the financial support of the China Scholarship Council (CSC). trainer (resp. trainee) has full authority on the trainee's (resp. trainer's) device and on the slave. When $0<\alpha<1$, both users share the slave control with a dominance (over the other user) which is function of $\alpha$. This control authority, shared between both users, is chosen according to their relative level of skills and experience. It determines the extent to which the motion of the slave tool depends on their individual commands.

The CLC architecture proposed by Khademian et al. in [1] provides to both users a shared control over the slave and a shared feedback from the environment according to $\alpha$. However, this architecture is modeled as a continuous linear time invariant system. As, in practice, the device dynamics and the tool environment induce nonlinear and unknown behaviors, its robustness may not be guaranteed. The other schemes found in the scientific literature (for instance, MECT [1] and Razi's gearbox [5]) provide a common control authority concept regardless differences in the controller realization. In [4], two different dominance factors are proposed to adjust the control authority over the slave and between users, and the stability is ensured by a Lyapunov function even in presence of delays and transmission packet drops. Still with the same authority mechanism, a robust higher order sliding mode control is proposed by [6] under unknown constant time delay.

The requirements for supervised training impose to help the trainee to train on the correct motions but also on the right level of force to provide. The aforementioned systems are mainly designed to share the control between two users so they feed back to each user a mix within the motions of the other user and of the slave. In these cases, both users only experience a portion tool motion or interaction with its environment. Under these circumstances, the trainee cannot acquire a full knowledge of the correct gestures. We aim to develop an architecture which guarantees full feedback to both users. This is where the scheme detailed in this paper differs from these works.

Precise requirements and objectives of our research project have already been detailed in [7] where an automatic authority switching mechanism has been introduced, and in [8] which features an adaptive authority mechanism. These two papers assume a passive behavior of both users and environment. In practice, this assumption cannot be guaranteed as both users and the environment can inject additional energy into the system. This is why we detail in this paper the design principles of this architecture and we introduce a passivity controller which prevents any passivity violation 




Fig. 1. Energy Shared Control (ESC) Dual-user system (the authority sharing mechanism is located inside the inner dashed box).

in real-time. Additionally this paper features a comparative study enabling to position our architecture with respect to aforementioned CLC and MECT architectures. This study assumes no delay in the loop as masters and slave interfaces are supposed to be controlled from the same computer in the same room. The introduction of delays will be introduced in future publications.

This paper is organized as follows. In section II, we present our dual-user system architecture. Section III-A introduces the metrics to evaluate position tracking performance used in section III and this latter details a comparative study. Section IV depicts validating experiments.

\section{Energy Shared Control Based Dual-User ARCHITECTURE}

This section details the design of a passive dual-user architecture. We chose to use an energy approach as it already provided interesting results in many teleoperation studies (such as in [9], [10], [11]). A dual-user system being a teleoperation system with a second user, this approach provides theoretical tools which help in adding this second user in the loop without increasing excessively the complexity of the study. Moreover, the adding of new extra users is also conceivable. The design of this architecture started from an entirely dual-user intrinsically passive system detailed in subsections II-A and II-B. Yet, this architecture was not able to provide full slave feedback to both users. This is why we enhanced it (see subsection II-C) to fulfill this purpose. Subsection II-D details how we guarantee the global passivity of this architecture.

\section{A. Authority Sharing Mechanism}

Inspired by the CLC architecture stated in [1], we propose a dual-user system based on Energy Shared Control (ESC), as shown in Fig. 1 (the blue part can be ignored in this section, it will be introduced in the next section). The authority sharing mechanism, based on parameter $\alpha$, is located in the inner dashed box: it is composed of three linear
Multiple-Input-Multiple-Output (MIMO) subsystems (Dirac systems as defined by Van Der Shaft et al in [12], whose representation are $\mathcal{D}_{m_{1}}, \mathcal{D}_{m_{2}}$ and $\mathcal{D}_{s}$ ) which, all together, redirect the energy flows (conveyed by power port variables $\left(\dot{\theta}_{i}, T_{i}\right.$ with $\left.i \in\left\{r_{1}, r_{2}, s_{1}, s_{2}, r_{s}\right\}\right)$ where $\dot{\theta}$ represents velocity signals and $T_{i}$ torque signals, visible in Fig. 1) between both users and the slave tool. Three dominance factors $\alpha, \beta_{1}, \beta_{2} \in[0,1]$ are introduced to achieve the goal of shared control. The $\mathcal{D}_{m_{1}}, \mathcal{D}_{m_{2}}$ and $\mathcal{D}_{s}$ relations are provided in Eq. $(2)^{1}$.

As their internal interconnection matrix is skewsymmetric, each subsystem $\left(\mathcal{D}_{m_{1}}, \mathcal{D}_{m_{2}}\right.$ and $\left.\mathcal{D}_{s}\right)$ is a powerconserving interconnection. This means that they are passive and lossless. The power-conserving interconnection of these three subsystems is then also passive. For example, considering the power balance of $\mathcal{D}_{m_{1}}$, we have,

$$
T_{r_{1}}^{T} \dot{\theta}_{r_{1}}+T_{s_{1}}^{T} \dot{\theta}_{s_{1}}+T_{s f_{1}}^{T} \dot{\theta}_{s f_{1}}=0
$$

which indicates the passivity.

$$
\begin{aligned}
\mathcal{D}_{m_{1}}:\left(\begin{array}{c}
\dot{\theta}_{r_{1}} \\
T_{s_{1}} \\
T_{s_{1}}
\end{array}\right)=\left(\begin{array}{ccc}
0 & \alpha & 1-\alpha \\
-\alpha & 0 & 0 \\
\alpha-1 & 0 & 0
\end{array}\right)\left(\begin{array}{l}
T_{r_{1}} \\
\dot{\theta}_{s_{1}} \\
\dot{\theta}_{s f_{1}}
\end{array}\right) \\
\mathcal{D}_{m_{2}}:\left(\begin{array}{c}
\dot{\theta}_{r_{2}} \\
T_{s_{2}} \\
T_{s f_{2}}
\end{array}\right)=\left(\begin{array}{ccc}
0 & 1-\alpha & \alpha \\
\alpha-1 & 0 & 0 \\
-\alpha & 0 & 0
\end{array}\right)\left(\begin{array}{l}
T_{r_{2}} \\
\dot{\theta}_{s_{2}} \\
\dot{\theta}_{s f_{2}}
\end{array}\right) \\
\mathcal{D}_{s}:\left(\begin{array}{c}
\dot{\theta}_{s_{1}} \\
\dot{\theta}_{s_{2}} \\
T_{r_{s}}
\end{array}\right)=\left(\begin{array}{ccc}
0 & 0 & \beta_{1} \\
0 & 0 & 1-\beta_{2} \\
-\beta_{1} & \beta_{2}-1 & 0
\end{array}\right)\left(\begin{array}{l}
T_{s_{1}} \\
T_{s_{2}} \\
\dot{\theta}_{r_{s}}
\end{array}\right)
\end{aligned}
$$

The relationship between $\alpha$ and $\beta_{1}, \beta_{2}$ are defined as,

$$
\beta_{1}=\left\{\begin{array}{lr}
\alpha, \quad \alpha=1,0 \\
1, & 0<\alpha<1
\end{array} \quad \beta_{2}=\left\{\begin{array}{lr}
\alpha, & \alpha=1,0 \\
0, & 0<\alpha<1
\end{array}\right.\right.
$$

\footnotetext{
${ }^{1}$ The notations of time-varying variables with $*(t)$ are abridged for simplification. The variables $\left(\dot{\theta}_{i}, T_{i}\right), i \in\left\{s f_{1}, s f_{2}\right\}$ belong to the blue part are discussed in the following section.
} 
Notice the reason by introducing $\beta_{1}, \beta_{2}$ is avoiding to repeat shared authority distribution at $\mathcal{D}_{s}$, since the shared power flow is already formed at $\mathcal{D}_{m_{1}}$ and $\mathcal{D}_{m 2}$. The function of $\mathcal{D}_{s}$ is to sum up the forces to the slave and deliver the velocities to both users. Regarding different control authority, we obtain three modes by changing the dominance factor $\alpha$ : training mode $(\alpha=1)$, guidance mode $(0<\alpha<1)$ and evaluation mode $(\alpha=0)$.

\section{B. Adding Compliance}

The previous core architecture does only redirect signals between the three power ports, it does not permit to control the end interfaces (Master 1, Master 2 and Slave). For this purpose, we inserted three Intrinsically Passive Controllers (IPC) (resp. IPC M1, IPC M2 and IPC S) in between them. The IPC controllers, introduced in [13] have the property to ensure a passive interaction of the controlled system with an unknown environment. Any robot model error may degrade the performances of the system but not its passivity. Thus, they provide a robust and powerful solution to control associated devices (Master 1, 2 and Slave). Therefore, their linking through power-conserving interconnections, with the passive core dual-user architecture composes a new passive system whatever controllers parameters and devices characteristics. This provides a natural robustness and leaves freedom to tune all IPC parameters for performance and global transparency. Moreover, IPC controllers provide a natural compliance which helps in training tasks. Thus, we obtain an intrinsically passive dual-user system, which corresponds to the outer dash-dot box in Fig. 1 (without the blue elements).

The only problem of this architecture is visible when $\alpha=$ 1 (resp. 0); in this case, the trainer (resp. trainee) exchanges all his energy exclusively with the slave. The system acts as a classical teleoperation system and completely isolates the trainee (resp. trainer) who can neither control the slave nor get feedback from it, which is opposite to the training requirements stipulating that the trainee (resp. trainer) should be able at least to observe the trainer (resp. trainee) motions.

\section{Providing Full Feedback to Both User}

In order to compensate for this problem, we added two identical modulated flow sources (MFS), associated with the velocity signal $\dot{\theta}_{r_{s}}$, defined in Eq. 7 in the following section and visible in blue in Fig. 1. The aim of the two flow sources is to feed back the complementary motion information of the slave which is missing in the core passive dual-user architecture. Consequently, both users experience a full motion feedback regardless of the control authority (i.e. the value of $\alpha$ ). This is checked by introducing $\dot{\theta}_{s f_{i}}=\dot{\theta}_{r_{s}}$ $(i \in\{1 ; 2\})$ into Eq. 7 :

$$
\begin{aligned}
& \dot{\theta}_{r_{1}}=\alpha \dot{\theta}_{s_{1}}+(1-\alpha) \dot{\theta}_{s f_{1}}=\alpha \dot{\theta}_{r_{s}}+(1-\alpha) \dot{\theta}_{r_{s}}=\dot{\theta}_{r_{s}} \\
& \dot{\theta}_{r_{2}}=(1-\alpha) \dot{\theta}_{s_{2}}+\alpha \dot{\theta}_{s f_{2}}=(1-\alpha) \dot{\theta}_{r_{s}}+\alpha \dot{\theta}_{r_{s}}=\dot{\theta}_{r_{s}}
\end{aligned}
$$

This way, thanks to IPC properties, when $\alpha=1, T_{h_{1}} \rightarrow$ $T_{e}$ and $T_{h_{2}} \rightarrow T_{e}$ when the trainee pushes his master interface so that $\theta_{m_{2}} \rightarrow \theta_{m_{1}}{ }^{2}$.

\footnotetext{
${ }^{2}$ Due to space limits, this property could not be detailed but the reader can see an illustration in section IV-A.
}

It is worth to mention that additional energy may be injected into the system by the modulated flow sources. This could violate the passivity of the whole architecture which may cause unstable behaviors.

\section{Passivity of the System}

Every part of the dual-chain (masters and slave robots controlled by the IPC controllers and energy shared control based architecture) being passive as stated in previous subsection, as long as both modulated flow sources hold passive behaviors, the passivity of the close-loop dual-user teleoperation system will be preserved. According to the definition of passivity in [12], the two MFS should only be able to extract energy from the dual-user chain (by the way, from the masters and the slave), which is translated by:

$$
\int_{u=0}^{u=t}\left(-T_{s f_{1}}^{T} \dot{\theta}_{s f_{1}}-T_{s f_{2}}^{T} \dot{\theta}_{s f_{2}}\right) \mathrm{d} u \geq 0, \forall t \geq 0
$$

Otherwise, the two MSF could inject extra energy into the system and potentially destabilize it. By integrating Eq. 2 and knowing that $\dot{\theta}_{s f_{1}}=\dot{\theta}_{s f_{2}}=\dot{\theta}_{r_{s}}$, the above passivity condition can be rewritten as,

$$
E_{p}=\int_{u=0}^{u=t}\left[(1-\alpha) T_{r_{1}}+\alpha T_{r_{2}}\right]^{T} \dot{\theta}_{r_{s}} \mathrm{~d} u \geq 0, \forall t \geq 0
$$

\section{E. Passivity Controller}

In practice, the passivity condition of Eq. 6 must always be satisfied. So, we use the following control laws for the modulated flow sources:

$$
\dot{\theta}_{s f_{1}}=\dot{\theta}_{s f_{2}}= \begin{cases}\dot{\theta}_{r_{s}}, & \text { if } E_{p} \geq 0 \\ 0, & \text { otherwise }\end{cases}
$$

The passivity condition in Eq. 6 can be considered as an energy storage function. As long as it is not empty, both MFS will be activated providing full feedback to both users. Otherwise, both MFS are deactivated, forcing the modulated flows $\dot{\theta}_{s f_{1}}$ and $\dot{\theta}_{s f_{2}}$ to 0 until the condition becomes checked again. This controller always guarantees the global passivity and may only deteriorate the additional feedback which means that in critical situations, the quality of feedback corresponds to the one depicted in section II-A.

\section{Comparative Study OF DuAL-USER ARCHITECTURES}

In this section, we propose to compare the Complementary Linear Combination (CLC) architecture, the Masters Correspondence with Environment Transfer (MECT) architecture [1] and our own one.

\section{A. Position Tracking Performance Criteria}

In the case of a dual-user training system, the position tracking errors between the users and the slave have been chosen to evaluate the different architectures. As it is an haptic system, force tracking can also be a good metrics but the different architectures tested in this study do not share the same policy in terms of force feedback (for instance, for CLC, $T_{h_{1}} \rightarrow \alpha T_{e}+(1-\alpha) T_{h_{2}}, T_{h_{2}} \rightarrow(1-\alpha) T_{e}+\alpha T_{h_{1}}$ 
while in MCET, $T_{h_{1}}$ and $T_{h_{2}} \rightarrow T_{e} / 2$ and, in ESC, $T_{h_{1}}$ and $T_{h_{2}} \rightarrow T_{e}$ ). An end-user study would be necessary to compare the effectiveness of all these systems in practice. This is why we limited this comparative study to motion tracking.

In free motion, the performance is evaluated through the tracking errors between the actual positions and the referenced ones, given by;

$$
\Phi_{i}=\left(\frac{1}{n} \sum_{k=1}^{n}\left\|\varepsilon_{i}(k)\right\|^{2}\right)^{\frac{1}{2}} \Delta_{i}=\left(\frac{1}{n} \sum_{k=1}^{n}\left\|\varepsilon_{i}(k)-\bar{\varepsilon}_{i}\right\|^{2}\right)^{\frac{1}{2}}
$$

where $\|\cdot\|$ is the Euclidean norm of $\mathbb{R}^{2} . \varepsilon_{i}(k)=$ $\left|\theta_{i}(k)-\theta_{i_{r}}(k)\right| /\left|\max \left(\theta_{i_{r}}\right)-\min \left(\theta_{i_{r}}\right)\right|, i \in\left\{m_{1}, m_{2}, s\right\}$ represents the relative error of the masters' and the slave's position $\left(\theta_{i}\right)$ against the amplitude of the reference trajectory $\left(\theta_{i_{r}}\right), \bar{\varepsilon}_{i}$ indicates the mean value of relative position errors and $n$ is the total number of samples of free motion positions. $\Phi_{i}$ reveals the position tracking preciseness, and $\Delta_{i}$ shows the standard deviation of position tracking errors. In case of perfect transparency, $\Phi_{i}=0$ and $\Delta_{i}=0$. Hence, a higher value indicates an undesirable performance.

\section{B. Trajectory Tracking Tasks}

To compare the three architectures on the same basis, we set the same sine wave based tracking task for each one. The task is carried out by simulated one d.o.f. joint mass robots as master and slave devices. Both the trainer and the trainee are modeled as damper-spring position-tracker systems. Regarding different control authority values, the task is defined as follows:

1) when $\alpha=1$, the trainer is the leader, and the trainee is the follower (i.e. training mode). They are modeled by

$$
T_{h_{1}}=500 \cdot\left(\theta_{m_{1 d}}-\theta_{m_{1}}\right)-10 \cdot \dot{\theta}_{m_{1}}, T_{h_{2}}=0
$$

where $\theta_{m_{1 d}}$ represents the position desired by the trainer $^{3}$. In this case, the reference trajectories (defined in Eq. 8) of both masters and the slave are given by:

$$
\theta_{m_{1 r}}=\theta_{m_{1 d}}, \quad \theta_{m_{2 r}}=\theta_{m_{1}}, \quad \theta_{m_{s r}}=\theta_{m_{1}}
$$

which means that the trainer tries to move the master 1 according to his desired trajectory while master 2 and the slave try to track the real motion of the trainer.

2) When $\alpha=0$, the trainee is the leader, while the trainer is the follower (i.e. evaluation mode). Their models become

$$
T_{h_{1}}=0, T_{h_{2}}=500 \cdot\left(\theta_{m_{2 d}}-\theta_{m_{2}}\right)-10 \cdot \dot{\theta}_{m_{2}}
$$

In this case, the reference trajectories of both masters and the slave are given by:

$$
\theta_{m_{1 r}}=\theta_{m_{2}}, \quad \theta_{m_{2 r}}=\theta_{m_{2 d}}, \quad \theta_{m_{s r}}=\theta_{m_{2}}
$$

\footnotetext{
${ }^{3}$ The next two cases follow the same sign convention. The parameters are chosen experimentally according to the tracking performance.
}

3) When $0<\alpha<1$, the trainer and the trainee are in shared control (i.e. guidance mode). Their models become then

$$
\left\{\begin{array}{l}
T_{h_{1}}=500 \cdot\left(\theta_{m_{1 d}}-\theta_{m_{1}}\right)-10 \cdot \dot{\theta}_{m_{1}} \\
T_{h_{2}}=500 \cdot\left(\theta_{m_{2 d}}-\theta_{m_{2}}\right)-10 \cdot \dot{\theta}_{m_{2}}
\end{array}\right.
$$

In this case, the reference trajectories of both masters and the slave are given by:

$$
\begin{aligned}
& \theta_{m_{1 r}}=\theta_{m_{1 d}}, \quad \theta_{m_{2 r}}=\theta_{m_{2 d}}, \\
& \theta_{m_{s r}}=\alpha \theta_{m_{1}}+(1-\alpha) \theta_{m_{2}}
\end{aligned}
$$

which shows that the slave tries to track the resulting shared motion of the two users, while the master 1 and master 2 try to track their respective desired trajectories.

The desired task trajectories of the trainer and the trainee are defined in Eq. 15. Notice that the differences between them are their amplitude and the situations when they are applied ( $\alpha$ values).

$$
\begin{aligned}
& \theta_{m_{1 d}}\{\alpha \in(0,1]\}= \begin{cases}\sin (t), & t \in[0,2 \pi] \\
0, & t \in[2 \pi, 10]\end{cases} \\
& \theta_{m_{2 d}}\{\alpha \in[0,1)\}= \begin{cases}0.8 \cdot \sin (t), & t \in[0,2 \pi] \\
0, & t \in[2 \pi, 10]\end{cases}
\end{aligned}
$$

TABLE I

PARAMETERS FOR EACH ARCHITECTURE

\begin{tabular}{|c||c|c|c|}
\hline \hline Scheme & Parameters & Unit & Value \\
\hline \multirow{4}{*}{$\mathrm{CLC}^{\mathrm{a}}(i=1,2)$} & $B_{m i}$ & $\mathrm{~N} \cdot \mathrm{s} / \mathrm{rad}^{2}$ & 1 \\
\cline { 2 - 4 } & $K_{m i}$ & $\mathrm{~N} / \mathrm{rad}^{2}$ & 50 \\
\cline { 2 - 4 } & $C_{5}$ & - & 0.2 \\
\hline \multirow{3}{*}{$\mathrm{MECT}^{\mathrm{b}}(i=1,2)$} & $C_{6 i}$ & - & 0.2 \\
\cline { 2 - 4 } & $B_{m i}$ & $\mathrm{~N} \cdot \mathrm{s} / \mathrm{rad}^{2}$ & 1 \\
\cline { 2 - 4 } & $K_{m i}$ & $\mathrm{~N} / \mathrm{rad}^{2}$ & 50 \\
\cline { 2 - 4 } & $C_{5}$ & - & 0.2 \\
\hline \multirow{3}{*}{$\mathrm{ESC}^{\mathrm{c}}$} & $C_{6 i}$ & - & 0.2 \\
\cline { 2 - 4 } & $k_{i_{m}}$ & $\mathrm{~N} / \mathrm{rad}^{2}$ & 200 \\
\cline { 2 - 4 } & $k_{c_{m}}$ & $\mathrm{~N} / \mathrm{rad}^{2}$ & 200 \\
\cline { 2 - 4 } & $k_{i_{s}}$ & $\mathrm{~N} / \mathrm{rad}^{2}$ & 100 \\
\cline { 2 - 4 } & $M_{c}$ & $\mathrm{~kg} / \mathrm{rad}^{2}$ & 0.0001 \\
\cline { 2 - 4 } & $M_{s}$ & $\mathrm{~kg} / \mathrm{rad}^{2}$ & 0.01 \\
\cline { 2 - 4 } & $b$ & $\mathrm{~N} \cdot \mathrm{s} / \mathrm{rad}^{2}$ & 0.8 \\
\cline { 2 - 4 } & $b_{s}$ & $\mathrm{~N} \cdot \mathrm{sad}^{2}$ & 0.2 \\
\hline \hline
\end{tabular}

${ }^{a, b}$ Notation, as in [1]. ${ }^{c}$ Notation, as in Figure 3.

\section{Tuning the Controller}

In the traditional bilateral teleoperation, stability and transparency are two conflicting control objectives. In order to compare the CLC, MCET and ESC architectures in the best situation, we have set their own parameters to guarantee their stability at first. Then, under the same excitation and parameters (same human force input signal, same control authority, etc.), we tuned the parameters of each controller to get their best position tracking performances. These parameters were then used in Matlab Simulink ${ }^{\circledR}$, in order to reproduce exactly the same stimuli for each scheme. Controllers parameters are given in Table I. 


\section{Comparison Over Different Control Authority Values}

We conducted a comparative study with different values of $\alpha$. The position tracking errors $\left(\Phi_{i}, \Delta_{i}\right)$ of each control framework are illustrated in Figure 2.
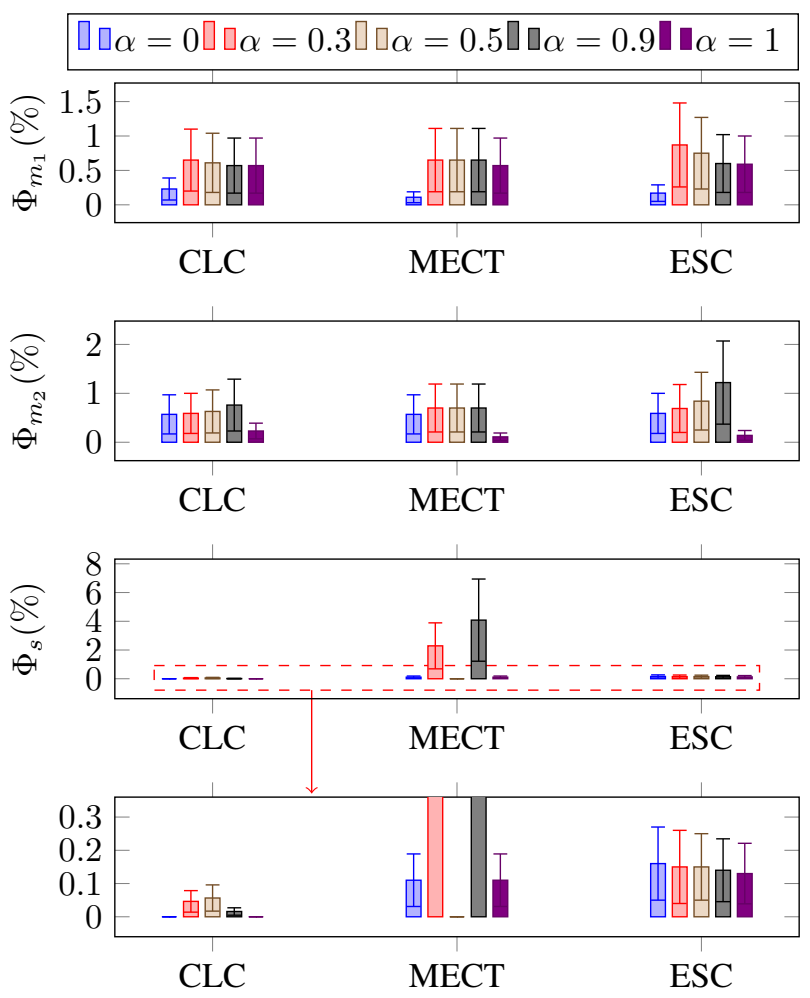

Fig. 2. Position tracking errors under different values of $\alpha$. The error bars indicate standard deviation errors $\left(\Delta_{i}, i=m_{1}, m_{2}, s\right)$. Note that subfigure 4 is the enlarged version of subfigure 3 .

For master 1 (trainer), we could conclude that the position tracking error is roughly the same with CLC, MECT and ESC for the same values of $\alpha$. More precisely, ESC presents a little higher error than the other two frameworks when $\alpha \in\{0.3 ; 0.5 ; 0.9\}$, but when $\alpha \in\{0 ; 1\}$, ESC gives slightly better results (for example, $\Phi_{m_{1}}=0.17 \%$ for ESC and $0.23 \%$ for CLC when $\alpha=0$ ).

It is worth to mention that the tracking error is globally inversely proportional to $\alpha$ when $0<\alpha<1$ in CLC and our schemes. It reveals that best performance is obtained by the user possessing most of the control authority, which is coherent with the general rule of this system. Similar results could be obtained for master 2 (trainee) due to the symmetry property of the system.

Considering the slave, good tracking performances are attained under CLC and our ESC framework despite the variations of control authority. It is worth to mention that the tracking error is significantly smaller when $\alpha \in\{0,1\}$ than when $0<\alpha<1$ for CLC framework (e.g., $\Phi_{m_{1}} \approx$ 0 when $\alpha=0,1)$. This reveals that the leader-follower mode (training/evaluation) outcomes better performance. The errors are almost the same within different authorities in our ESC architecture, yet good enough for tracking purpose with the error less than $0.2 \%$. Concerning the MECT framework, the best tracking performance is achieved when $\alpha=0.5$. It discloses that the MECT scheme is competitive only when $\alpha \in\{0 ; 0.5 ; 1\}$.

As a conclusion, this comparative study demonstrated that our ESC architecture has almost equal performance compared with CLC and MECT schemes, in common use cases (training/guidance/evaluation modes). These results are explained by the fact that only a portion of slave position signal is fed back to the users in CLC scheme, and a compensation part is formed from the other user, while in MECT scheme, there is no direct slave position signal feedback to either of the users (see [1]).

\section{EXPERIMENTS}

To evaluate the performances of this framework in practice, the following experiments have been conducted. The haptic training software part was built into MATLAB/Simulink as shown in Fig. 3. Two Sensable ${ }^{\circledR}$ PHANTOM Omni devices (only the rotation of their first joint the elbow - in the one-degree-of-freedom case) were used as the two masters operated by the trainer and the trainee. The library developed in [14] was used to communicate with MATLAB/Simulink. The devices' kinematic and dynamic parameters can be found in [15]. A simulated one d.o.f. joint mass robot was set up for the virtual slave. Notice that the slave IPC controller has a different form than the two masters ones, for reverse causality needs.

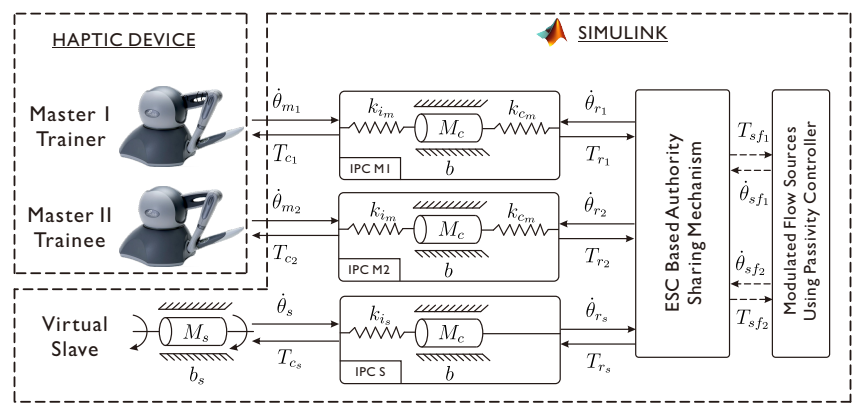

Fig. 3. The experimental setup

\section{A. Experimental Results}

The three different modes (training, guidance and evaluation) of the proposed ESC architecture have been experimented. A virtual wall, modeled as a spring and a damper in parallel, was set at angle $0.5 \mathrm{rad}$. The target position is at angle $0.3 \mathrm{rad}$. The hand torques were estimated by a Nicosia observer described in [16]. The tracking of positions and hand/environment torques are shown in Fig. 4. The experiment time is separated into six periods (from A to F), with corresponding parameter and properties given in Table II. From the figure, the trainer has full control authority during period $\mathrm{A}$ when he leads his device to the target position. Meanwhile, the trainee and the slave follow him (training mode). In phase B, the control authority is switched to the trainee for evaluation purpose; the slave and the trainer follow him. After then, in period C, both the trainer and the trainee experience a shared control over the slave where the trainer tries to guide the slave to the target position. 

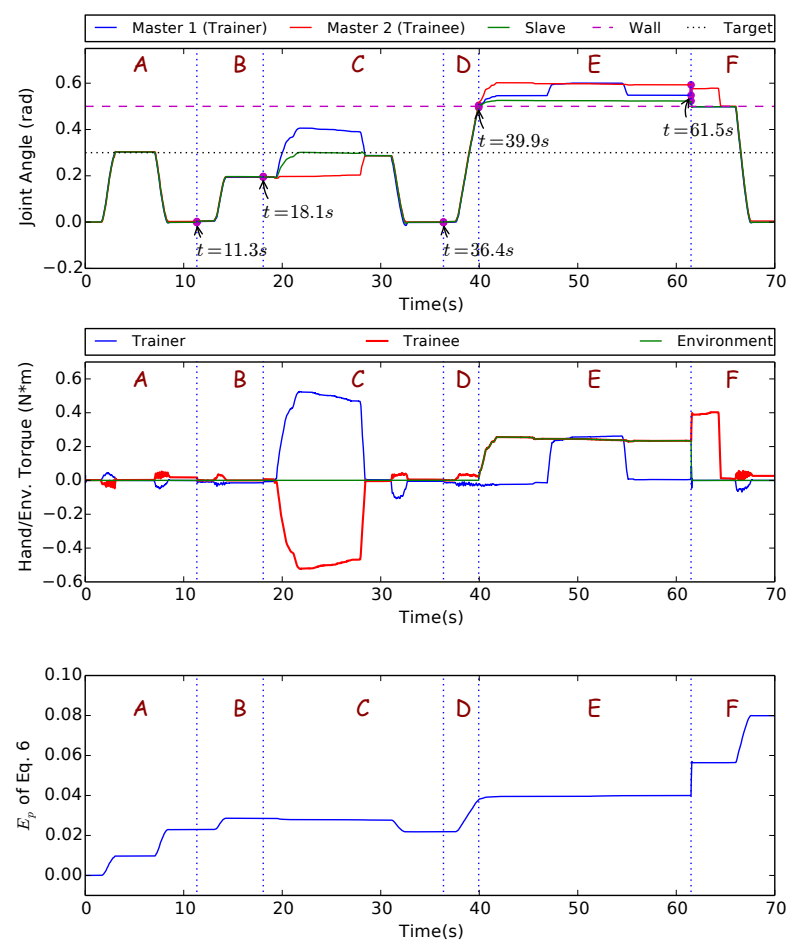

Fig. 4. The positions, torques tracking and energy passivity checking

TABLE II

THE EXPERIMENT PERIODS

\begin{tabular}{|c|c|c|c|c|c|c|}
\hline Period & A & B & C & D & E & F \\
\hline$\alpha$ & 1 & 0 & 0.5 & 0 & 0 & 1 \\
\hline Mode & T & E & G & E & E & T \\
\hline Wall Contact & $\mathrm{N}$ & $\mathrm{N}$ & $\mathrm{N}$ & $\mathrm{N}$ & $\mathrm{Y}$ & $\mathrm{N}$ \\
\hline
\end{tabular}

Wall Contact: $\mathrm{Y}=\mathrm{Yes}, \mathrm{N}=\mathrm{No}$

The trainee notices his own incorrect motion and then he leads his device to the target position as well. The full control authority is switched back to the trainee again in phase $\mathrm{D}$ during which he leads the slave up to the virtual wall. The slave collides the wall in period $\mathrm{E}$. The trainee feels the reaction torque from the environment. Note that, by default, in this situation, the trainer has no environment force feedback as $\alpha=0$. He needs to guide his device to the same position as the trainee's one to be able to feel the same reaction torque (visible during $47 \mathrm{~s}$ to $55 \mathrm{~s}$ time lapse). Note that, in contact period, as usual with many teleoperators, the masters' position is not exactly the same as the slave because of their own compliance. Stiffer masters would decrease this offset but could also introduce oscillations in free motion. On authority switching, in period $\mathrm{F}$, the slave moves back from the wall as the new leader (the trainer) does not provide enough torque at this moment to counter the previous wall reaction torque. Finally, the slave and the trainee follow the trainer's motion towards the initial position. Note the energy checking of Eq. 6 is shown in subfigure 3, which is always non-negative that ensures the passivity condition. The MFS are always activated to providing full slave motion feedback. To conclude, experiments demonstrate a shared control behavior and a good tracking performance in motion and forces.

\section{CONClusion}

In this paper, in order to provide a robust supervised hands-on training system, we designed a passive dual-user system. The passivity of the closed-loop system is proved and relies on a real-time passivity controller. A task based comparative study with CLC and MCET architectures from [1], has been conducted and proves relative good performances in terms of motion control. Real-time experiments exhibit a complete training scenario showing also good performances in terms of force (torque) feedback. Future work should provide a comparative study on the force tracking during restricted motions. Time-delays and multiple degrees of freedom will also be considered.

\section{REFERENCES}

[1] B. Khademian and K. Hashtrudi-Zaad. Shared control architectures for haptic training: performance and coupled stability analysis. The International Journal of Robotics Research, 30(13):1627-1642, Mar. 2011.

[2] Panait L., Akkary E., Bell R.L., Dudrick S.J. Roberts K.E., and Duffy A.J. The role of haptic feedback in laparoscopic simulation training. Journal of Surgical Research, 156(2):312-316, Oct. 2009.

[3] S.S. Nudehi, R. Mukherjee, and M. Ghodoussi. A shared-control approach to haptic interface design for minimally invasive telesurgical training. IEEE Transactions on Control Systems Technology, 13(4):588-592, Jul. 2005.

[4] A. Ghorbanian, S.M. Rezaei, A.R. Khoogar, M. Zareinejad, and K. Baghestan. A novel control framework for nonlinear time-delayed dual-master/single-slave teleoperation. ISA Transactions, 52(2):268277, Mar. 2013

[5] K. Razi and K. Hashtrudi-Zaad. Analysis of coupled stability in multilateral dual-user teleoperation systems. IEEE Transactions on Robotics, 30(3):631-641, Jun. 2014.

[6] H. Santacruz-Reyes, L.G. Garcia-Valdovinos, H. Jimenez-Hernandez, A.G. Lopez-Segovia, and O.A. Dominguez-Ramirez. Robust higher order sliding mode based impedance control for dual-user teleoperation under unknown constant time delay. In Electrical Engineering, Computing Science and Automatic Control (CCE), 2014 11th International Conference on, pages 1-6, Sept 2014.

[7] F. Liu, A. Lelevé, D. Eberard, and T. Redarce. A dual-user teleoperation system with online authority adjustment for haptic training. In Proceedings of 37th Annual International Conference of IEEE Engineering in Medicine and Biology Society (EMBC), Aug. 2015.

[8] F. Liu, A. Lelevé, D. Eberard, and T. Redarce. A dual-user teleoperation system with adaptive authority adjustement for haptic training. In Proceedings of 4th International Workshop on Medical and Service Robots, Jul. 2015.

[9] R. Anderson and M.W. Spong. Bilateral control of teleoperators with time delay. IEEE Transactions on Automatic Control, 34(5):494-501, May 1989.

[10] Dongjun Lee and P.Y. Li. Passive bilateral feedforward control of linear dynamically similar teleoperated manipulators. IEEE Transactions on Robotics and Automation, 19(3):443-456, Jun. 2003.

[11] Dongjun Lee and M.W. Spong. Passive bilateral teleoperation with constant time delay. IEEE Transactions on Robotics, 22(2):269-281, Apr. 2006.

[12] A. J. Van der Schaft. L2-Gain and Passivity Techniques in Nonlinear Control. Springer-Verlag London, 2000.

[13] S. Stramigioli. Modeling and ipc control of interactive mechanical systems: A coordinate-free approach. Springer, 2001.

[14] Carlos I. Aldana, Emmanuel Nu no, Luis Basa nez, and Eduardo Romero. Operational space consensus of multiple heterogeneous robots without velocity measurements. Journal of the Franklin Institute, 351(3):1517-1539, 2014.

[15] T. Sansanayuth, I. Nilkhamhang, and K. Tungpimolrat. Teleoperation with inverse dynamics control for phantom omni haptic device. In Proceedings of SICE Annual Conference (SICE), pages 2121-2126, Aug. 2012.

[16] S. Nicosia and P. Tomei. Robot control by using only joint position measurements. IEEE Transactions on Automatic Control, 35(9):10581061, Sep. 1990 